American Journal of Applied Sciences 6 (9): 1708-1713, 2009

ISSN 1546-9239

(C) 2009 Science Publications

\title{
An Effective Location-Based Power Conservation Scheme for Mobile Ad Hoc Networks
}

\author{
Khalid Kaabneh, Azmi Halasa and Hussein Al-Bahadili \\ Faculty of Information Systems and Technology, \\ The Arab Academy for Banking and Financial Sciences, Amman 11942, Jordan
}

\begin{abstract}
Problem statement: One of the major issues in current reactive routing protocols for Mobile Ad Hoc Networks (MANETs) is the high bandwidth and power consumptions during the routing process. In this study, we proposed and evaluated the performance of an efficient LocationBased Power Conservation (LBPC) scheme for MANETs. Approach: In this scheme, the transmitting node utilized the location-information of the first-hop neighbors to adjust its radio transmission range according to one of the following criteria: Farthest first-hop neighbor, average distance of the first-hop neighbors and a random distance between the nearest and the farthest first- hop neighbors. Results: A number of simulation were carried-out to evaluate the power conservation ratio that can be achieved for two route discovery algorithms, namely, pure flooding and Location-Aided Routing Scheme 1 (LAR-1) algorithms. Conclusion: The simulation results demonstrated that the scheme can provide power conservation ratios between $10-50 \%$ without adding any extra overheads or complexity to the routing algorithm.
\end{abstract}

Key words: MANETs, dynamic routing protocols, route discover, flooding optimization algorithms, LAR-1 algorithm, wireless power consumption

\section{INTRODUCTION}

A MANET is defined as a collection of low power, wireless, mobile nodes forming a temporary network without the aid of any established infrastructure or centralized administration ${ }^{[1-3]}$. In MANETs, a node usually consumes its power in wireless communication (in other words, message passing), message processing and other operation missions. It has been realized that the power consumed in message passing is orders of magnitude higher than what is consumed in other tasks; therefore, minimizing it is a vital requirement to extend the lifetime of the battery-powered nodes. Nodes are communicating with each other for two main objectives; these are: route establishment and data forwarding using certain routing protocols ${ }^{[3-5]}$.

The most widely used routing protocols in MANETs are known as reactive routing protocols, such as Dynamic Source Routing (DSR) ${ }^{[6]}$, Ad Hoc On Demand Distance Vector (AODV) ${ }^{[7]}$, Zone Touting Protocol $(\mathrm{ZRP})^{[8]}$ and Location-Aided Routing $(\mathrm{LAR})^{[9]}$. Reactive protocols mainly consist of two phases: route discovery and route maintenance ${ }^{[10]}$. The cost of information exchange (in terms of bandwidth and power consumption) during route discovery is higher than the cost of point-to-point data forwarding ${ }^{[5]}$.

A number of optimization algorithms have been developed to minimize bandwidth and power consumption during route discovery, such as: locationbased $^{[9]}$, probabilistic ${ }^{[1]}$, multipoint relaying ${ }^{[11]}$, counterbased $^{[12]}$, distance-based ${ }^{[9]}$, cluster-based schemes ${ }^{[13]}$. All algorithms are based on reducing the number of Retransmissions for the Route Request (RREQ) messages, so that the bandwidth is saved and contention, duplicate reception and node power consumption are reduced. These advantages may cost some reduction in the network reachability.

In this study, we propose and evaluate the performance of Location-Based Power Conservation (LBPC) scheme that can be used to reduce power consumption in MANETs. The scheme makes use of location-information to reduce power consumption, where it is assumed that each node within the network is always aware of the location of all other nodes within the network. With the significant advancement in Global Positioning System (GPS) technology and the drop in its costs, the nodes location may be provided by using a built-in GPS ${ }^{[9]}$.

Corresponding Author: Hussein Al-Bahadili, The Arab Academy for Banking and Financial Sciences, P.O. Box 13190, Amman 11942, Jordan Tel: +962-6-5502900 Fax: +962-6-5105176 
In this scheme, the node utilizes the locationinformation available on the first-hop neighbors to adjust its radio transmission range to cover an area up to: Farthest first-hop neighbor, average distance of the first-hop neighbors, or a random distance between the nearest- and farthest first-hop neighbors.

In this study, the power conservation ratios for two flooding algorithms, namely, pure flooding ${ }^{[1-3]}$ and LAR-1 ${ }^{[9]}$ algorithms, are evaluated. For the two algorithms, the effect of a number of network parameters, such as: node density, node mobility, node maximum radio transmission range, on the power conservation ratio, number of retransmission, average duplicate reception and reachability, are investigated using the MANET simulator (MANSim) ${ }^{[14,15]}$.

The simulation results demonstrated that the scheme can provide power conservation ratios that vary from $10-50 \%$ depending on the network parameters and the adjustment criteria. However, adjusting the radio transmission range to be equivalent to the average distance of the first-hop neighbors provides higher power conservation ratio, but it also slightly affects other network parameters.

Related work: A lot of work has been done to minimize power consumption in Ah Hoc networks and Wireless Sensor Networks (WSNs), where in such networks nodes are either fixed or has a very limited mobility. Therefore, they experience a very limited topology or route variations and most of the communication power consumption is exhausted for data communications. While in a MANET, due to the high nodes speeds, the network usually suffers from continuous topology and route variations, thereafter, nodes consume more power for route establishment. Reducing power consumption has been addressed through the adjustment of the node radio transmission range, which is done through different aspects till now.

Ingelrest et $a l^{[16]}$ investigated the problem of minimum energy broadcasting in Ah Hoc networks. Two localized broadcasting protocols were presented, based on derived "target" radius, these are: (i) the target radius Local Minimum Spanning Tree (LMST) Broadcast Oriented Protocol (TR-LBOP). It computes the minimal radius needed for connectivity and increases it up to the target one after applying a neighbor elimination scheme on a reduced subset of direct neighbors. (ii) The Target Radius and Dominating Sets-Based (TRDS) protocol, each node first considers only neighbors whose distance is not greater than the target radius; and neighbors in a localized connected topological structure such as Relative Neighborhood Graph (RNG) or LMST.
Jan Blumenthal et al. ${ }^{[17]}$ investigated that the autonomous localization of nodes in WSNs is essential to minimize the complex self-organization task consequently and to enhance network lifetime. A method to measure the distance using the minimal transmission power between a transmitting node and a receiving node was proposed. They showed that the determined distance was very precise and has a low variance. Dongjin et al. ${ }^{[18]}$ investigated the impact of variable transmission power on link quality and proposed variable power link quality control techniques to enhance the performance of data delivery in WSNs.

Pan et al. ${ }^{[19]}$ considered a two-tiered WSN consisting of sensor clusters deployed around strategic locations and Base-Stations (BSs) whose locations are relatively flexible. Within a sensor cluster, there are many Small Sensor Nodes (SNs) that capture, encode and transmit relevant information to the designated area and there is at least one Application Node (AN) that receives raw data from these $\mathrm{SNs}$, creates a comprehensive local-view and forwards the composite bit-stream toward a BS. They focused on the Topology Control (TC) process for ANs and BSs, which constitute the upper tier of a two-tiered WSN. They proposed approaches to maximize the topological network lifetime of the WSN, by arranging BS location and inter-a relaying optimally. They evaluated the performance of TC and proved that its efficiency is as a vital process to maximize the lifetime of WSNs.

Lui and $\mathrm{Li}^{[20]}$ considered the problem of TC in a network of heterogeneous wireless devices with different maximum transmission ranges, where asymmetric wireless links are not uncommon. For such environment, they presented a distributed TC algorithm to calculate the per-node minimum transmission power, so that (1) reachability between any two nodes is guaranteed to be the same as in the initial topology: And (2) nodal transmission power is minimized to cover the least number of surrounding nodes. Their simulation results demonstrated the correctness and effectiveness of the proposed algorithm.

Zarifzadeh et al. ${ }^{[21]}$ redefined the problem of TC regarding both transmission range and traffic load parameters. They mathematically formulated a mixed integer linear programming problem to find optimal solutions. Then, they introduced polynomial-time heuristic algorithms to practically solve the problem. During construction of network topology, they deliberately took into account the impact of the employed routing method on load of individual nodes. They showed the advantages of their proposed algorithms through simulations. 
Wireless communication power consumption model: Wireless communication power consumption depends on two factors, these are: the radio transmission range and data transmission rate. For a node transmitting a bit-stream at a rate of $b$ bps over a distance $r \mathrm{~m}$, the minimal transmitter power consumption $\mathrm{E}_{\mathrm{t}}$, for freespace radio communication, is given by:

$\mathrm{E}_{\mathrm{t}}(\mathrm{b}, \mathrm{r})=\left(\alpha_{1}+\alpha_{2} \mathrm{r}^{2}\right) \mathrm{b}$

Where:

$\alpha_{1}=$ The power consumed in the transmitter electronic circuit for message processing, which is the distance-independent power consumption term

$\alpha_{2}=$ The power consumed in message passing, which is the distance-dependent power consumption term

Equation 1 is applicable even with a more complicated model (e.g., including multi-path fading and geographical shadowing effects), as long as the distance related power consumption can be isolated empirically.

For a node to receive a bit-stream at a rate of $\mathrm{b}$ bps from other node, the power consumed in the receiver circuit is given by:

$\mathrm{E}_{\mathrm{r}}(\mathrm{b})=\beta \mathrm{b}$

Thus, the total power consumed by a node to bypass and forward a bit-stream at a rate $\mathrm{b}$ bps over a distance $r \mathrm{~m}$, is given by:

$E(b, r)=\left[\left(\alpha_{1}+\alpha_{2} r^{2}\right)+\beta\right] b$

In a MANET that consists of $\mathrm{N}$ nodes, the route discovery using, for example, pure flooding involves $\mathrm{n}$ transmissions and $\mathrm{m}$ receptions. Thus, the power consumed in message passing and processing $\left(\mathrm{E}_{\mathrm{f}}\right)$ is expressed as:

$E_{f}=n E_{t}(b, r)+m E_{r}(b)$

Substituting Eq. 1 and 2 into Eq. 4 yields:

$E_{f}=n\left(\alpha_{1}+\alpha_{2} r^{2}\right) b+m \beta b$

It is clear from the above equation that the total power consumption during route discovery depends on four parameters, these are: Number of retransmissions $(\mathrm{n})$, number of receptions (m), data transmission rate (b) and transmission distance (r). However, due to the fact that the power consumption in message processing is very small as compared to the power consumption in message passing, then Eq. 5 can be simplified to:
$\mathrm{E}_{\mathrm{f}}=\alpha_{2} \mathrm{nb} \mathrm{r^{2 }}$

The proposed LBPC scheme: In what follows, we present a description of the proposed LBPC scheme. It makes use of location information to reduce power consumption during route discovery in MANETs. In this scheme, it is assumed that each node is aware of the location of all other nodes within the network and the nodes location that is used may be provided by a builtin GPS ${ }^{[9]}$. The LBPC scheme can be summarized as follows:

Each node searches for all first-hop neighbors:

- Calculate the Euclid distance (r) between the node and all other nodes within the network

- Compare $r$ with the maximum radio transmission range $\left(R_{\max }\right)$ of the node. If $r \leq R_{\max }$, then this node is a first-hop neighbor, otherwise it is not

Adjust the radio transmission Rage (R) according to one of the following criteria:

- Maximum distance $\left(\mathrm{r}_{\max }\right)$, which means the transmission will reach the farthest first-hop neighbor, i.e., set $R=r_{\max }$

- Average distance ( $\mathrm{r}_{\text {avg }}$ ) and its associated standard deviation $(\sigma)$, i.e., set $R=r_{\text {avg }} \pm \sigma$. However, in this work we use $\mathrm{R}=\mathrm{r}_{\text {avg }}$

- Random distance ( $\mathrm{r}_{\mathrm{ran}}$ ) lies between the nearest and farthest nodes, which is calculated as: $r_{\text {ran }}=r_{\min }+$ $\left(r_{\max }-r_{\min }\right) \xi$, where $r_{\min }$ and $r_{\max }$ are the distances of the nearest and farthest nodes, respectively and $\xi$ is a random number between zero and one $(0 \leq \xi<1)$, i.e., set $R=r_{\text {ran }}$

The criterion that is used for adjusting the transmission range may affect other network parameters, such as the number of retransmission, average duplicate reception, reachability ${ }^{[22]}$. However, it can be seen that none of these parameters will be affected if $R=r_{\max }$. The efficiency of the proposed scheme is defined in terms of a power conservation ratio $\left(\mathrm{P}_{\mathrm{c}}\right)$, which is calculated as:

$P_{c}=\left(1-\frac{\hat{n} R^{2}}{\bar{n} R_{\text {max }}^{2}}\right) \times 100$

Where:

$\hat{n}$ and $\bar{n} \quad=$ The number of retransmission with and without implementing the power conservation scheme, respectively 
$\mathrm{R}$ and $\mathrm{R}_{\max }=$ The adjusted and maximum radio transmission range, respectively

In a MANET that uses LAR algorithm, each node is already aware of the location of other nodes within the network. Therefore, this scheme can be implemented in such networks with absolutely no added overheads. In a network that uses other routing algorithms and in order to minimize communication overheads, nodes can be configured to exchange its location with their first-hop neighbors only.

\section{RESULTS}

In order to evaluate and analyze the performance of the LBPC scheme, three scenarios were simulated using MANSim $^{[15]}$. These scenarios evaluate the effects of a number of input network parameters, such as: node density $\left(\mathrm{n}_{\mathrm{d}}\right)$, node mobility $(\mathrm{u})$ and node maximum radio transmission range $\left(\mathrm{R}_{\max }\right)$, respectively. The performance is evaluated in terms of $\mathrm{P}_{c}$, number of Retransmission (RET), Average Duplicate Reception (ADR) and Reachability $(\mathrm{RCH})$, for the three radio transmission range adjustment criterion (e.g., maximum, farthest, average).

In this study, the simulations were carried-out for two route discovery algorithms: pure flooding and LAR-1. The input parameters for these three scenarios are shown in Table 1. The simulation results are shown in Table 2-4.

Table 1: Input parameters for the three scenarios

\begin{tabular}{|c|c|c|c|}
\hline Parameters & Scenario \# 1 & Scenario \# 2 & Scenario \# 3 \\
\hline Node distribution & Random & Random & Random \\
\hline Network area & $600 \times 600 \mathrm{~m}$ & $600 \times 600 \mathrm{~m}$ & $600 \times 600 \mathrm{~m}$ \\
\hline Number of nodes (n) & $50,75,100$ nodes & 100 nodes & 100 nodes \\
\hline Transmission radius $\left(\mathrm{R}_{\max }\right)$ & $100 \mathrm{~m}$ & $100 \mathrm{~m}$ & $100,125,150 \mathrm{~m}$ \\
\hline Average node speed $(\mathrm{u})$ & $2 \mathrm{~m} \mathrm{sec}^{-1}$ & $\begin{array}{l}2,4 \text { and } 6 \\
\mathrm{~m} \mathrm{sec}^{-1}\end{array}$ & $2 \mathrm{~m} \mathrm{sec}^{-1}$ \\
\hline Simulation time $\left(\mathrm{T}_{\text {sim }}\right)$ & \multicolumn{3}{|c|}{$\begin{array}{l}1200,600 \text { and } 300 \mathrm{sec} \text { for } 50,75 \text { and } 100 \text { nodes, } \\
\text { respectively }\end{array}$} \\
\hline Pause time $(\tau)$ & \multicolumn{3}{|l|}{$\tau=\left(0.75 \times \mathrm{R}_{\max }\right) \mathrm{u}^{-1}$} \\
\hline
\end{tabular}

Table 2: Results for scenario \#1: Investigate the effect of the node density $\left(\mathrm{n}_{\mathrm{d}}\right)$

\begin{tabular}{|c|c|c|c|c|c|c|c|c|}
\hline \multirow[b]{2}{*}{$\mathrm{n}$} & \multicolumn{2}{|l|}{ RET } & \multicolumn{2}{|l|}{ ADR } & \multicolumn{2}{|l|}{$\mathrm{RCH}$} & \multicolumn{2}{|l|}{$\mathrm{P}_{\mathrm{c}}$} \\
\hline & $\mathrm{R}_{\max } / \mathrm{R}_{\mathrm{far}}$ & $\mathrm{R}_{\mathrm{avg}}$ & $\mathrm{R}_{\max } / \mathrm{R}_{\mathrm{far}}$ & $\mathrm{R}_{\mathrm{avg}}$ & $\mathrm{R}_{\text {max }} / \mathrm{R}_{\mathrm{far}}$ & $\mathrm{R}_{\mathrm{avg}}$ & $\mathrm{R}_{\mathrm{far}}$ & $\mathrm{R}_{\mathrm{avg}}$ \\
\hline \multicolumn{9}{|c|}{ Pure flooding } \\
\hline 50 & 0.605 & 0.585 & 3.477 & 3.136 & 0.620 & 0.598 & 25.8 & 40.0 \\
\hline 75 & 0.793 & 0.861 & 7.267 & 6.456 & 0.806 & 0.875 & 17.6 & 20.4 \\
\hline 100 & 0.929 & 0.900 & 10.789 & 9.513 & 0.939 & 0.911 & 13.6 & 26.2 \\
\hline \multicolumn{9}{|c|}{ LAR-1 } \\
\hline 50 & 0.129 & 0.093 & 0.274 & 0.158 & 0.475 & 0.305 & 22.5 & 51.3 \\
\hline 75 & 0.142 & 0.125 & 0.405 & 0.272 & 0.615 & 0.530 & 16.5 & 35.3 \\
\hline 100 & 0.167 & 0.162 & 0.622 & 0.549 & 0.819 & 0.737 & 11.7 & 26.0 \\
\hline
\end{tabular}

Table 3: Results for scenario \#2: Investigate the effect of the node mobility or node speed (u)

\begin{tabular}{|c|c|c|c|c|c|c|c|c|}
\hline \multirow[b]{2}{*}{$\mathrm{u}$} & \multicolumn{2}{|l|}{ RET } & \multicolumn{2}{|l|}{ ADR } & \multicolumn{2}{|l|}{$\mathrm{RCH}$} & \multicolumn{2}{|l|}{$\mathrm{P}_{\mathrm{c}}$} \\
\hline & $\mathrm{R}_{\max } / \mathrm{R}_{\mathrm{far}}$ & $\mathbf{R}_{\text {avg }}$ & $\mathrm{R}_{\max } / \mathrm{R}_{\mathrm{far}}$ & $\mathbf{R}_{\mathrm{avg}}$ & $\mathrm{R}_{\max } / \mathrm{R}_{\mathrm{far}}$ & $\mathrm{R}_{\mathrm{avg}}$ & $\mathrm{R}_{\mathrm{far}}$ & $\mathrm{R}_{\mathrm{ave}}$ \\
\hline \multicolumn{9}{|c|}{ Pure flooding } \\
\hline 2 & 0.929 & 0.900 & 10.789 & 9.513 & 0.939 & 0.911 & 13.6 & 26.2 \\
\hline 4 & 0.901 & 0.903 & 9.305 & 8.305 & 0.911 & 0.915 & 14.3 & 24.1 \\
\hline 6 & 0.911 & 0.896 & 8.692 & 7.778 & 0.921 & 0.908 & 14.4 & 26.0 \\
\hline \multicolumn{9}{|c|}{ LAR-1 } \\
\hline 2 & 0.167 & 0.162 & 0.622 & 0.549 & 0.819 & 0.737 & 11.7 & 26.0 \\
\hline 4 & 0.157 & 0.143 & 0.490 & 0.427 & 0.762 & 0.640 & 13.0 & 31.6 \\
\hline 6 & 0.154 & 0.137 & 0.445 & 0.367 & 0.743 & 0.611 & 13.0 & 33.3 \\
\hline
\end{tabular}

Table 4: Results for scenario \#3: Investigate the effect of the node transmission range $\left(\mathrm{R}_{\max }\right)$

\begin{tabular}{|c|c|c|c|c|c|c|c|c|}
\hline \multirow[b]{2}{*}{$\mathrm{R}_{\max }$} & \multicolumn{2}{|l|}{ RET } & \multicolumn{2}{|l|}{ ADR } & \multicolumn{2}{|l|}{$\mathrm{RCH}$} & \multicolumn{2}{|l|}{$\mathrm{P}_{\mathrm{c}}$} \\
\hline & $\mathrm{R}_{\max } / \mathrm{R}_{\mathrm{far}}$ & $\mathrm{R}_{\mathrm{avg}}$ & $\mathrm{R}_{\max } / \mathrm{R}_{\mathrm{far}}$ & $\mathrm{R}_{\mathrm{avg}}$ & $\mathrm{R}_{\max } / \mathrm{R}_{\mathrm{far}}$ & $\mathrm{R}_{\mathrm{avg}}$ & $\mathrm{R}_{\mathrm{far}}$ & $\mathrm{R}_{\mathrm{ave}}$ \\
\hline \multicolumn{9}{|c|}{ Pure flooding } \\
\hline 100 & 0.929 & 0.900 & 10.789 & 9.513 & 0.939 & 0.911 & 13.6 & 26.2 \\
\hline 125 & 0.987 & 0.977 & 11.033 & 9.320 & 0.997 & 0.987 & 10.8 & 24.0 \\
\hline 150 & 0.990 & 0.990 & 15.111 & 12.280 & 1.000 & 1.000 & 8.7 & 23.3 \\
\hline \multicolumn{9}{|c|}{ LAR-1 } \\
\hline 100 & 0.167 & 0.162 & 0.622 & 0.549 & 0.819 & 0.737 & 11.7 & 26.0 \\
\hline 125 & 0.166 & 0.158 & 0.575 & 0.497 & 0.835 & 0.746 & 10.5 & 26.6 \\
\hline 150 & 0.184 & 0.182 & 0.761 & 0.623 & 0.917 & 0.876 & 6.8 & 24.2 \\
\hline
\end{tabular}




\section{DISCUSSION}

The results show that the LBPC scheme provides a $\mathrm{P}_{\mathrm{c}}$ that varies between 10 to $50 \%$ depending on the input network parameters. For similar parameters, adjusting node radio transmission range to cover a distance equivalent to the average distances of the firsthop neighbors yields higher $P_{c}$ than adjusting the transmission range to reach farthest first-hop neighbor, but at the same time it affects RET, ADR and RCH. In what follows the results obtained for each scenario are summarized and briefly discussed.

The results for scenario \#1 are shown in Table 2. It shows that as node density increases from 50-100 nodes, $\mathrm{P}_{\mathrm{c}}$ is decreased, for both pure flooding and LAR1 algorithms. Also, it can be realized that pure flooding provides higher $\mathrm{P}_{c}$ than LAR-1 for equivalent node density.

For scenario \# 2, the results in Table 3 show that the node mobility has insignificant effect on $\mathrm{P}_{c}$ for both algorithms. For Scenario \# 3, $P_{c}$ decreases as $R_{\max }$ is increased from 100-150 m, for both algorithms and all other parameters are remained unchanged. This is because the number of retransmissions is increased and nodes consume more power during these retransmissions than what they save by radio transmission range adjustment.

\section{CONCLUSION}

This study presents an efficient Location-Based Power Conservation (LBPC) scheme for MANETs. The scheme utilizes the location information of the node first-hop neighbors to adjust the radio transmission range of the transmitting node to cover (i) an area up to the farthest first-hop neighbor, (ii) an area at a radius equivalent to the average distance the first-hop neighbors and (iii) an area up to a random distance between the nearest and the farthest first-hop neighbors.

Three scenarios have been simulated to evaluate the power conservation ratios for two route discovery algorithms: pure flooding and LAR-1. The simulations show that a power conservation ratio of $10-50 \%$ can be achieved depending on the network environment and the simulation time. This is considered as a very encouraging result as it can be achieved without added overheads or complexity. However, the results obtained demonstrate that the power conservation is reduced as node density increases and all other network parameters are remained unchanged. The same is for increasing radio transmission range. While increasing node mobility has little effect on the power conservation ratio.

\section{REFERENCES}

1. Bani-Yassein, M., M.O. Khaoua, L.M. Mackenzie and S. Papanastasiou, 2006. Performance analysis of adjusted probabilistic broadcasting in mobile $\mathrm{Ad}$ Hoc networks. Int. J. Wireless Inform. Networks, 13: 127-140. DOI: 10.1007/s10776-006-0027-0

2. Scott, D. and A. Yasinsac, 2004. Dynamic probabilistic retransmission in Ad Hoc networks. Proceedings of the International Confernece on Wireless Networks, (ICWN'04), pp: 158-164. http://citeseerx.ist.psu.edu/viewdoc/summary?doi= 10.1.1.85.9638

3. Sasson, Y., D. Cavin and A. Schiper, 2003. Probabilistic broadcast for flooding in wireless mobile Ad Hoc networks. Proceedings of the IEEE Conference on Wireless Communication and Networking, Mar. 20-20, IEEE Xplore Press, USA., $\quad$ pp: $1124-1130 . \quad$ DOI: 10.1109/WCNC.2003.1200529

4. Tseng, S., S. Ni, Y. Chen and J. Sheu, 2002. The broadcast storm problem in a mobile Ah Hoc network. J. Wireless Networks, 8: 153-167. http://portal.acm.org/citation.cfm?id=506905

5. Rahman, A., W. Olesinski and P. Gburzynski, 2004. Controlled flooding in wireless Ah Hoc networks. Proceedings of IEEE International Workshop on Wireless Ad Hoc Networks, May 31June 03, IEEE Xplore Press, USA., pp: 73-78. DOI: 10.1109/IWWAN.2004.1525544

6. Johnson, D.B., Y. Hu and D. Maltz, 2007. The dynamic source routing (DSR) protocol for mobile Ah Hoc networks for IPv4. RFC 4728, MANET Working Group, IETF. http://www.ietf.org/rfc/rfc4728.txt.

7. Perkins, C. and E. Belding-Royer and S. Das, 2003. Ad-hoc on-demand distance vector (AODV) routing. RFC 3561, MANET Working Group, IETF. http://tools.ietf.org/rfc3561.

8. Haas, Z., M. Pearlman and P. Samar, 2002. The zone routing protocol (ZRP) for Ah Hoc networks. MANET Working Group, IETF.

http://tools.ietf.org/id/draft-ietf-manet-zone-zrp-04.txt

9. Ko, Y.B. and N.H. Vaidya, 2000. Location-aided Routing (LAR) in mobile Ad Hoc networks. Wireless Networks, 6: 307-321. http://portal.acm.org/citation.cfm?id=352164

10. Royer, E. and C. Toh, 1999. A review of current routing protocols for $\mathrm{Ah}$ Hoc mobile wireless networks. IEEE Person. Commun. Mag., 6: 46-55. http://citeseerx.ist.psu.edu/viewdoc/summary?doi= 10.1.1.41.3409 
11. Qayyum, A., L. Viennot and A. Laouiti, 2002. Multipoint relaying for flooding broadcast messages in mobile wireless networks. Proceedings of the 35th Hawaii Int. Conf. on System Sciences, June 7-10, IEEE Xplore Press, USA., pp: 298-307. http://ieeexplore.ieee.org/xpl/freeabs_all.jsp?arnum ber $=994521$

12. Tseng, Y., S. Ni and E. Shih, 2003. Adaptive approaches to relieving broadcast storm in a wireless multihop mobile Ad Hoc network. IEEE Trans. Comput., 52: 545-557. DOI: 10.1109/TC.2003.1197122

13. Wu, J. and W. Lou, 2003. Forward-node-set-based broadcast in clustered mobile Ad Hoc networks. Wireless Networks Mobile Comput., 3: 155-173. http://cat.inist.fr/?aModele $=$ afficheN\&cpsidt $=1469$ 7289

14. Al-Bahadili, H., O. Al-Basheer and A. Al-Thaher, 2007. A location aided routing-probabilistic algorithm for flooding optimization in MANETs. Proceedings of Mosharaka International Conference on Communications, Networking and Information Technology, Mar. 19-22, AmmanJordan.

http://www.cs.columbia.edu/ hgs/bib/net07.bib

15. Al-Bahadili, H., 2009. On the Use of DiscreteEvent Simulation in Computer Networks Analysis and Design. In: Handbook of Research on Discrete-Event Simulation Environments: Technologies and Applications, Abu-Taieh, E.M.O. and A.A. El-Sheikh (Eds.). Information Science Reference, ISBN: 978-1-60566774-4, pp: 673 .

16. Ingelrest, F., D. Simplot-Ryl and I. Stojmenovic, 2006. Optimal transmission radius for energy efficient broadcasting protocols in Ad Hoc and sensor networks. IEEE Trans. Parall. Distribut. Syst., 17: 536-547. DOI: 10.1109/TPDS.2006.74

17. Blumenthal, J., F. Reichenbach and D. Timmermann, 2006. Minimal transmission power vs. signal strength as distance estimation for localization in wireless sensor networks. Proceedings of the 3rd IEEE Int. Workshop on Wireless Ad Hoc and Sensor Networks, Sept. 28-28, IEEE Xplore Press, Reston, VA., pp: 761-766. DOI: 10.1109/SAHCN.2006.288558
18. Son, D., B. Krishnamachari and J. Heidemann, 2004. Experimental study of the effects of transmission power control and blacklisting in wireless sensor networks. Proceedings of the 1st IEEE Conf. on Sensor and Ad Hoc Communication and Networks, Oct. 4-7, IEEE Xplore Press, USA., pp: 289-298. DOI: 10.1109/SAHCN.2004.1381929

19. Pan, J., Y.T. Hou, L. Cai, Y. Shi and S.X. Shen, 2003. Topology control for wireless sensor networks. Proceedings of 9th Annual International Conference on Mobile Computing and Networking, Sept. 14-19, San Diego, CA, USA., pp: 286-299. http://portal.acm.org/citation.cfm?id=939015

20. Liu, J. and B. Li, 2003. Distributed topology control in wireless sensor networks with asymmetric links. Proceedings of the IEEE Global Telecommunications Conference, Dec. 1-5, IEEE Xplore Press, USA., pp: 1257-1262. DOI: 10.1109/GLOCOM.2003.1258440

21. Zarifzadeh, S., A. Nayyeri and N. Yazdani, 2008. Efficient construction of network topology to conserve energy in wireless Ah Hoc networks. J. Comput. Commun., 31: 160-173. http://portal.acm.org/citation.cfm?id=1327694

22. Al-Bahadili, H. and Y. Jaradat, 2007. Development and performance analysis of a probabilistic flooding in noisy mobile Ad Hoc networks. Proceedings of the 1st International Conference on Digital Communications and Computer Applications, (DCCA'07), Jordan, pp: 1306-1316. http://www.cis.just.edu.jo/dcca2007 